\section{Going immune on beige fat}

\section{By Kai-Jye Lou, Senior Writer}

Since its founding in 2010, Ember Therapeutics Inc. has been exploiting the biology of brown and beige fat to treat metabolic diseases, including obesity and type 2 diabetes. Now, the biotech has an option to license the hormone meteorin glial cell differentiation regulator-like, one of the first molecules found to activate a newly identified immune systemregulated pathway responsible for driving the formation of beige fat., ${ }^{1,2}$

Beige adipocytes and their brown adipocyte cousins are mitochondria-rich cells that metabolize triglycerides to generate heat. Beige adipocytes are found interspersed within white adipose tissue, whereas brown adipocytes are found in concentrated depots but only at trace levels in adults. Beige and brown adipocytes are more metabolically active than white adipocytes, which store energy as triglycerides.

Various stimuli, such as cold exposure, are known to promote the formation of beige adipocytes in white fat-a process known as browning.

Antiobesity therapeutics based on increasing the amount of brown fat have received more attention from industry as the biology and regulation of brown fat are better understood than that of beige fat. ${ }^{3}$

New data concurrently published in a pair of studies in Cell show that the immune system is a key regulator of beige fat generation and thermogenesis. The findings could spark efforts to develop therapies that focus on increasing the amount of beige fat.

"Brown adipose tissue is well innervated by sympathetic neurons, and past studies have already demonstrated the role of the sympathetic nervous system in regulating its formation and activity," said Ember CSO Jasbir Seehra. "White adipose tissue, on the other hand, has less innervation, so the lingering question prior to these two papers has been how white adipose tissue gets stimulated to form beige fat."

In one study, a group at the University of California, San Francisco led by Ajay Chawla teased out the components of the pathway by which cold exposure drives the development of beige fat. Chawla is an associate professor at the Cardiovascular Research Institute at UCSF.

The UCSF researchers used a series of knockout mice to show that cold exposure-induced formation of beige fat depends on eosinophils, IL-4 (BSF1) and IL-13 signaling, the downstream transcription factor signal transducer and activator of transcription 6 (STAT6) and monocyte recruitment to adipose tissues via CC chemokine receptor 2 (CCR2; CD192) signaling.

The recruited monocytes differentiate into macrophages and are activated by IL-4 and IL-13. Macrophage activation increases the expression of tyrosine hydroxylase, which is the key rate-limiting enzyme that catalyzes catecholamine synthesis (see Figure 1, "Pathway for driving beige fat formation"). Catecholamines induce thermogenesis activity and the generation of beige adipocytes in white adipose tissue.

In mice with diet-induced obesity, i.p. injection of Il-4 increased beige fat mass and decreased total body mass and fat mass compared with vehicle injection. In the obese mice, Il-4 also improved several measures of obesity-related metabolic dysfunction such as glucose homeostasis and insulin sensitivity.

"We've identified a core mechanism

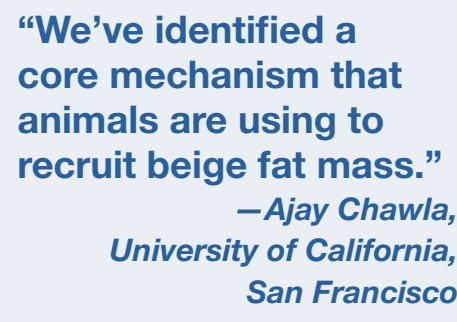
that animals are using to recruit beige fat mass," said Chawla.

In the other study, a group led by Bruce Spiegelman identified meteorin glial cell differentiation regulator-like (METRNL) as a key mediator of the heat-generating activity of beige fat. In mice, an adenoviral vector that induced overexpression of Metrnl in the liver increased thermogenesis from beige fat and decreased whole-body fat content compared with a control vector. Metrnl overexpression also induced the expression of multiple anti-inflammatory genes.

Spiegelman is a professor of cell biology and medicine at the Dana-Farber Cancer Institute and Harvard Medical School. He is a cofounder of Ember and chair of its scientific advisory board.

In a mouse model of obesity, vector-induced Metrnl overexpression improved glucose tolerance and increased whole-body energy expenditure.

Analyses of mouse adipose tissues suggested that Metrnl induces the generation of beige adipocytes in white adipose tissue and thermogenesis activity via the same pathway described by Chawla's group.

Chawla noted that although the identified molecules themselves are probably not yet ready for therapeutic development, the findings "do suggest that one may want to engage in more rigorous studies to understand the described pathway and then move toward the development of new antiobesity therapies."

"The reported findings represent major steps toward the realization of a therapeutic that works by increasing beige fat," added Seehra.

Ember already is developing molecules that promote the formation of brown fat and/or the browning of white fat.

Seehra said that Ember is interested in METRNL but declined to say whether the company has active programs related to the hormone.

\section{Figuring out therapies}

The new data could spur efforts to investigate other immune system components as therapeutics that promote the development of beige fat.

"Figuring out whether there are specific immune components that could serve as targets for therapy opens up an interesting new area," said Patrick Seale, an assistant professor in the Department of Cell and Developmental Biology at the Perelman School of Medicine at the University of Pennsylvania. "Understanding the types of immune cells and how they are regulated in fat will now be important." Seale said 
Figure 1. Pathway for driving beige fat formation. Cold stimulus promotes beige fat formation and thermogenesis, but the pathways involved in such processes were unclear. The recently hypothesized immune system-mediated pathway for driving these processes could yield new ways to treat obesity, type 2 diabetes and related metabolic disorders.

Through this pathway, cold stimulus triggers recruitment of CC chemokine receptor 2 (CCR2; CD192)+ monocytes to white adipose tissues, where they differentiate into macrophages. Cold stimulus also induces eosinophils in those tissues to release type 2 cytokines, such as IL-4 (BSF1) and IL-13 (small yellow circles).

The cytokines induce alternative activation of macrophages. This process leads to increased expression of tyrosine hydroxylase in myeloid cell populations found in white adipose tissues, including the macrophages themselves.

The increased expression of tyrosine hydroxylase results in more secretion of catecholamines (small purple circles) from myeloid cells, which in turn drives the formation of beige adipocytes and increases their thermogenesis activity.

Whether these beige adipocytes are formed from white adipocytes or some precursor cell population in white adipose tissue has not been determined.

At least two points of intervention (red dashed arrows) have been identified. As reported in Rao et al., the hormone meteorin glial cell differentiation regulator-like (METRNL) (gray circles) can induce expression of IL-4 and IL-13 and promote beige fat formation and thermogenesis activity. Separately, Qiu et al. report that infusion of IL-4 itself has similar effects.

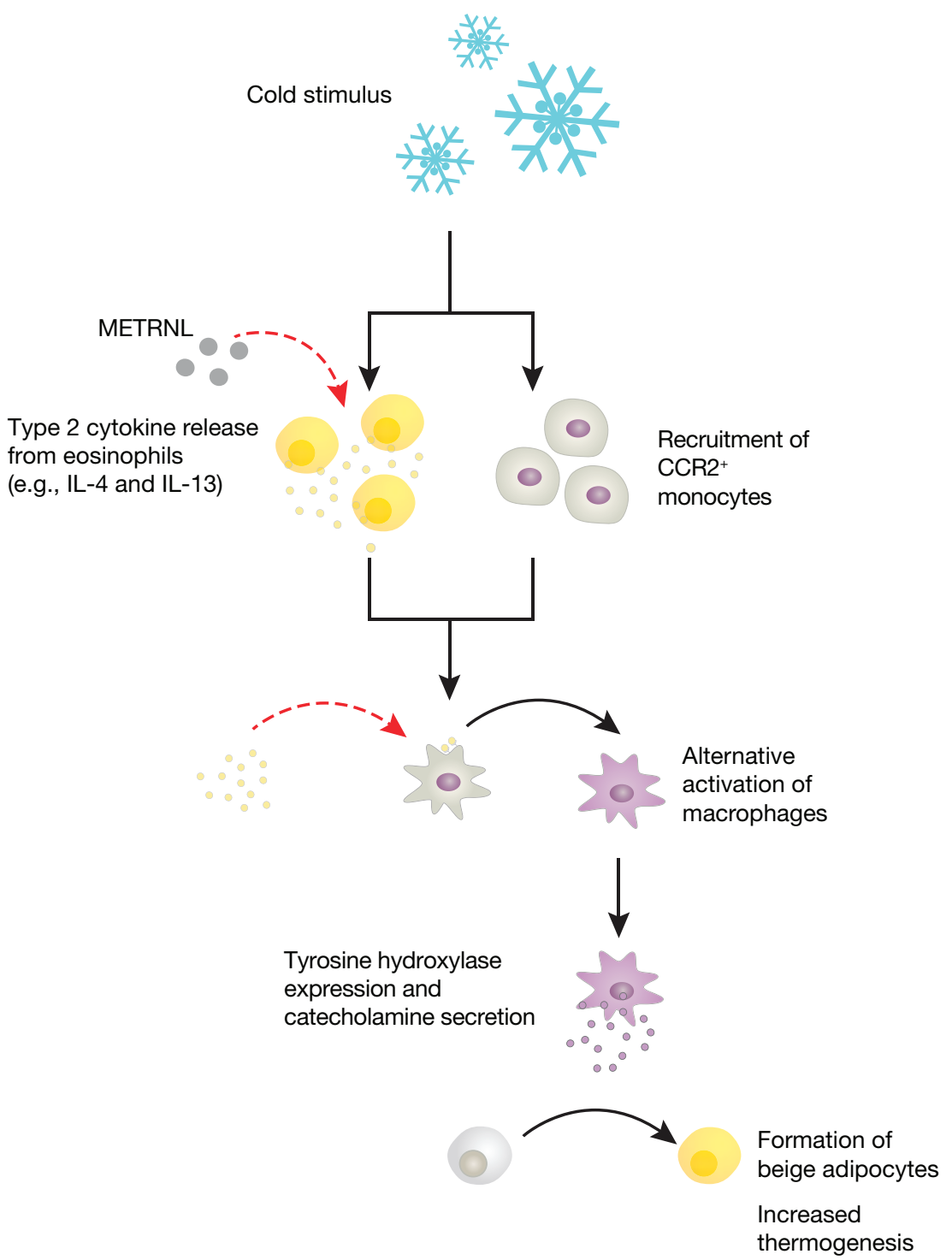

that previous efforts to promote beige fat development have primarily focused on manipulating the fat cells themselves or targeting CNS pathways.

"What these new studies describe is an entirely new pathway that promotes the formation of beige adipocytes, and I think every step of this pathway should be reviewed for potential therapeutic purpose," said Sven Enerbäck, a professor of medical genetics at the University of Gothenburg and a member of Ember's scientific advisory board.

He said that it will be important to determine the extent to which the activated macrophages could maintain catecholamine production and to find the identity of the downstream

"Understanding the types
of immune cells and how
they are regulated in fat
will now be important."
-Patrick Seale,
Perelman School of Medicine at
the University of Pennsylvania

needs to be addressed is: can you modulate the pathway in a manner that does not have adverse outcomes?"

Plutzky is director of The Vascular Disease Prevention Program at Brigham and Women's Hospital. He also is an associate professor at Harvard Medical School and a member of Ember's scientific advisory board.

Spiegelman said that it appears the components of the newly described pathway could be manipulated to promote beige fat development, but he added that it is too early to say whether such manipulation could be achieved in a manner that mitigates potential side effects.

The tractability of METRNL and IL-4 as catecholamine-activated precursor cells that give rise to beige adipocytes.

"These studies provide an excellent starting point for the development of new therapies but also raise questions on what the signaling pathway could be doing in other tissues," said Jorge Plutzky. "The question that drugs in their own right also remains unclear.

"A fair amount of work still needs to be done to understand how [METRNL] works at the molecular level before one can come up with a form of the protein with the appropriate pharmacokinetic properties to allow it to be developed as a therapeutic," said Seehra. "Until you know 


\section{ANALYSIS}

what the receptors are, coming up with molecular forms of [METRNL] that could be delivered therapeutically is challenging."

Seale added that it will be

"The reported findings represent major steps toward the realization of a therapeutic that works by increasing beige fat." - Jasbir Seehra, Ember Therapeutics Inc. important to determine how METRNL expression is regulated in fat and muscle tissues.

Spiegelman's group is trying to identify the receptor for METRNL. As the hormone also showed an anti-inflammatory effect, his group is evaluating whether METRNL could have utility in other diseases such as exercise-induced muscle damage and muscular dystrophy.

As for IL-4, Chawla noted that it might be difficult to develop the cytokine as a therapy for obesity and related metabolic conditions because it regulates many aspects of immunity, such as B cell and $\mathrm{T}$ cell proliferation.

"You probably wouldn't want to have some of these aspects chronically turned on," Chawla told SciBX. He noted that if antiobesity strategies based on IL-4 were to be pursued, "you will want to develop something that is selective, short-lived and targeted to the specific tissue."

Chawla said that his group is conducting studies to identify additional cell types and signaling networks that could be important to the described pathway for regulating beige fat. He added that it also will be important for others to develop and evaluate proof-ofconcept molecules that target various points of the pathway.

Dana-Farber has filed a patent application covering the results related to METRNL. The findings described by the UCSF group are unpatented.

Lou, K.-J. SciBX 7(26); doi:10.1038/scibx.2014.753

Published online July 10, 2014

\section{REFERENCES}

1. Rao, R.R. et al. Cell; published online June 5, 2014; doi:10.1016/j.cell.2014.03.065

Contact: Bruce Spiegelman, Dana-Farber Cancer Institute, Boston, Mass.

e-mail: bruce_spiegelman@dfci.harvard.edu

2. Qiu, Y. et al. Cell; published online June 5, 2014; doi:10.1016/j.cell.2014.03.066

Contact: Ajay Chawla, University of California, San Francisco, Calif. e-mail: ajay.chawla@ucsf.edu

3. Harms, M. \& Seale, P. Nat. Med. 19, 1252-1263 (2013)

\section{COMPANIES AND INSTITUTIONS MENTIONED}

Brigham and Women's Hospital, Boston, Mass.

Dana-Farber Cancer Institute, Boston, Mass.

Ember Therapeutics Inc., Boston, Mass.

Harvard Medical School, Boston, Mass.

Perelman School of Medicine at the University of Pennsylvania, Philadelphia, $\mathrm{Pa}$.

University of California, San Francisco, Calif.

University of Gothenburg, Gothenburg, Sweden 